\section{OP ENACTING PPI IN QUALITATIVE MENTAL HEALTH RESEARCH: PEER RESEARCHER INVOLVEMENT IN DATA ANALYSIS IN THE 'CORE' STUDY}

N Morant, ${ }^{*}$ B Lloyd-Evans, CORE project team led by S. Johnson. Division of Psychiatry, University College London, UK

*Presenting author.

\subsection{6/bmjopen-2015-UCLSymposiumAbstracts.4}

Patient and public involvement (PPI) is now an expected component of UK publicly-funded health research. Compatibilities with PPI aims and values mean that qualitative research is often a vehicle for enacting PPI. This presentation focuses on the involvement of "peer researchers" (service users and carers) in qualitative data analysis, and offers critical reflections of the practicalities of this process in the context of mental health research and an academic-led project.

"CORE" (Crisis resolution team Optimisation and RElapse prevention) is a large NIHR-funded research project that aims to optimise the functioning of crisis resolution teams (CRTs). CRTs provide intensive home-based treatment for people experiencing an acute mental health crisis, as an alternative to hospital admission. They exist in all NHS Mental Health Trusts in England, but their performance and effectiveness is variable. The CORE project adopts a collaborative model of service user and carer involvement within an academic-led project. A large service user and carer working group contributes to a range of project activities including a qualitative study of stakeholders' views on current and best CRT practice, in which peer researchers have conducted interviews and been involved in data analysis.

Faced with a large data set of more than 100 pieces of data, and little existing guidance on peer researcher involvement in qualitative data analysis, we attempted to develop an approach that enabled genuine involvement and maximised the methodological benefits of collaboration. Additional aims were to build research skills and capacity, and to obtain feedback from participants about their experiences of this. Peer researcher involvement began in the early stages of thematic analysis, in order to maximise contributions to the development of thematic codes at the point when these were most fluid and open. This was part of a staged process that meshed the work and perspectives of peer researchers with those of the academic researcher team. The presentation will describe this process and the rationale behind it, and discuss critical issues such as whether academic-led collaborations perpetuate or have the capacity to challenge existing power inequalities that are greater in mental health than other healthcare contexts. 